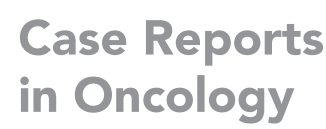

Case Reports

in Oncology

This article is licensed under the Creative Commons Attribution-NonCommercial 4.0 International License

\title{
Disease Response to Pazopanib in Follicular Dendritic Cell Sarcoma
}

\author{
Pooja Shah ${ }^{a}$ Smit Shah $^{a}$ Nicole Agostinob \\ aDepartment of Internal Medicine, Reading Hospital, Tower Health, West Reading, PA, USA; \\ ${ }^{b}$ Department of Hematology/Oncology, Reading Hospital, Tower Health, West Reading, PA, \\ USA
}

\section{Keywords}

Follicular dendritic cell sarcoma $\cdot$ Pazopanib $\cdot$ Kinase inhibitors $\cdot$ Pembrolizumab

\begin{abstract}
Follicular dendritic cell sarcoma (FDCS) is a rare sarcoma, which commonly presents as a slowgrowing, painless mass. There are only a few hundred reported FDCS cases, and the role for adjuvant chemo- or radiation therapy has not been established. Choosing an appropriate therapy in disseminated disease can therefore be challenging. A 26 -year-old patient with FDCS was admitted with dyspnea, fever, and night sweats. He was found to have a large right hemothorax with compressive atelectasis on initial imaging. CT of the chest revealed multiple bilateral lung and pleural nodules with associated bilateral hilar adenopathy, a hypodense mass within the right hemithorax, and necrotic right external iliac and inguinal nodes. Inguinal node biopsy diagnosed FDCS. The patient was initially treated with cyclophosphamide, doxorubicin, vincristine, and prednisone chemotherapy. Gemcitabine/Taxotere was given as second-line therapy and pembrolizumab as third-line therapy, with continued disease progression after 2 cycles of both regimens. The patient was switched to fourth-line therapy with pazopanib and had a partial response for 9 months. This case illustrates a successful FDCS treatment with pazopanib. Due to the rarity of FDCS, where large studies comparing treatment approaches are not available, recommendations for optimal treatment are not well defined. This case is in support of growing evidence suggesting that FDCS responds to systemic therapies that are used for soft tissue sarcoma, such as pazopanib.
\end{abstract}




\section{Introduction}

Follicular dendritic cell sarcoma (FDCS) is an uncommon sarcoma which most commonly presents as a slow-growing, painless mass. FDCS may easily be confused with other solid tumors. This rare sarcoma was first described by Monda's group in 1986 and is now recognized to be a low-grade sarcoma of mesenchymal dendritic cell origin. FDCS originates in antigen-presenting cells of the B-cell follicles of lymph nodes. Dendritic cells play a crucial role in the immune system, contributing to both phagocytosis and antigen processing and presentation to B and T cells [1]. FDCS has been found to have an immunophenotype distinct from other malignant histiocytic tumors. FDCS is associated with widespread chromosomal instability, dysregulation of cell cycle progression, nuclear factor- $\kappa B$ activation, mitogen-activated protein kinase activation, and immune evasion. The mitotic index of FDCS is usually low $(0-10)$, and the Ki-67 is most often less than $25 \%$. FDCS characteristically has a significant infiltrate of $\mathrm{B}$ and $\mathrm{T}$ cells, including $\mathrm{T}$ lymphocytes that express terminal deoxytransferase. The diagnosis of FDCS is based on tissue pathology and presence of immunohistologic markers of dendritic cells (i.e., CD21, CD23, CD35, serglycin, and follicular dendritic cell-secreted protein). FDCS has histologic features of a low-grade sarcoma, with spindle-shaped cells that contain weakly eosinophilic cytoplasm seen in a storiform or whorled pattern [2]. There have only been a few hundred reported cases of FDCS, and most cases seen have presented with localized disease. However, extranodal sites such as tonsils, lung, liver, or spleen have been described. Males and females are affected equally, and the mean age of incidence is in the fifth decade of life, although reported cases have ranged from 9 to 82 years of age [3]. Unfavorable prognostic factors include large tumors $(>6 \mathrm{~cm})$, significant cellular atypia, coagulative necrosis seen on pathologic slides, intra-abdominal location, and high mitotic rate $(>5$ mitoses in 10 high-power field) [4].

\section{Case Presentation}

We report a 26-year-old patient with FDCS. The patient was admitted in September of 2018 with dyspnea, fever, and night sweats and was found to have a large right hemothorax with compressive atelectasis on initial imaging. CT chest revealed multiple bilateral lung and pleural nodules with associated bilateral hilar adenopathy, a thick-walled septated centrally hypodense mass within the right hemithorax, and necrotic right external iliac and inguinal nodes. Initial biopsy at our institution was negative for malignancy. Second pathology opinion at tertiary care center diagnosed FDCS. One month later, the patient was admitted for recurrent malignant right pleural effusion and underwent a VATS procedure. The VATS procedure demonstrated significant tumor infiltration and ingrowth within the entire pleural cavity. The patient was initially treated with cyclophosphamide, doxorubicin, vincristine, and prednisone (CHOP) chemotherapy. After 2 cycles, CT scan showed disease progression. Next generation sequencing did not show any actionable targets. Microsatellite instability was stable, and tumor mutation burden remained low. PD-L1 was negative $2+, 1 \%$. Gemcitabine and Taxotere chemotherapy were given as second-line treatment. After 2 cycles, he once again had disease progression in all anatomic areas. Most concerning was a lytic lesion in the right femur that was a risk for pathologic fracture. He was initiated on radiation therapy to the right femur and pembrolizumab simultaneously. After 2 cycles of pembrolizumab, he had disease progression in all areas of disease except the radiation field (Fig. 1). The patient was switched to fourth-line therapy with pazopanib $800 \mathrm{mg}$ daily. After $1 \mathrm{month}$ of pazopanib, he had significant symptomatic improvement in his cough. He had improved exercise tolerance and decreased oxygen requirements. After 2 months of pazopanib, the patient experienced at

\section{Karger'}


Fig. 1. CT chest with contrast status after pembrolizumab (03/2019), continued disease progression.

Fig. 2. CT chest with contrast status after 2 months of pazopanib $(05 / 2019)$, significant disease regression.
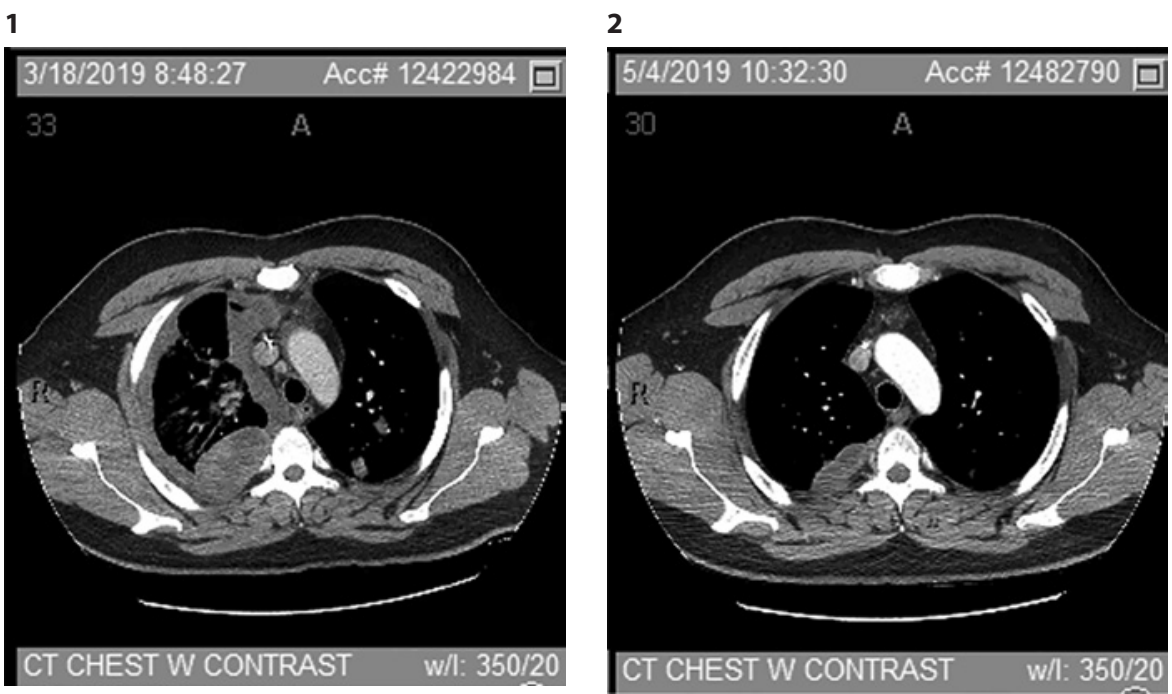

50-75\% partial disease response (Fig. 2). A partial response was sustained for 9 months before disease progression occurred in the lungs. After pazopanib, the patient received sunitinib, ifosfamide/carboplatin/etoposide chemotherapy and cabozantinib with short interval progression on all therapies. At the time of this reporting, hospice discussions are taking place.

\section{Discussion}

Due to the rarity of FDCS, large studies comparing treatment approaches are not available, and thus recommendations for optimal treatment are not well defined. The primary treatment of localized FDCS is surgical resection. A role for adjuvant therapy has not been established for either radiation therapy or chemotherapy [5]. FDCS usually has an indolent course, but $50 \%$ of patients develop local recurrence. An aggressive course is possible as well, with metastases to the lung, liver, and lymph nodes. Growing evidence suggests that FDCS responds to systemic therapies that are used for soft tissue sarcoma.

Lymphoma regimens such as CHOP and ABVD have been used to treat FDCS with variable outcomes. Other lymphoma-based regimens such as ifosfamide, carboplatin, and etoposide (ICE) have also been employed in cases of FDCS with outcomes similar to CHOP [6]. A case review of 50 cases by Wang et al. [7] showed no recurrence of disease in $80 \%$ patients in which CHOP therapy was used after excision of the sarcoma.

Sarcoma-based regimens such as gemcitabine and Taxotere as well as Adriamycin, ifosfamide, and mesna have also been used with success in FDCS. There have been several case reports of FDCS that show that therapy with gemcitabine in combination with a taxane (paclitaxel or docetaxel) have been successful [6]. A study of 46 patients with FDCS in which different chemotherapy agents were used, gemcitabine and Taxotere combination therapy showed the best outcomes. Among patients who received gemcitabine and Taxotere with/ without other modalities, $42 \%$ (5/12) were complete responders, $41 \%$ were partial responders (5/12), and 2 patients were non-responders. In the 10 responders, 6 patients had surgery prior to chemotherapy, 3 had radiotherapy, and 1 had chemotherapy alone [8].

A second study of 66 patients with FDCS who were treated with chemotherapy (gemcitabine and a taxane, CHOP-based, or ifosfamide-based) in non-resectable disease, showed an overall response rate of $80 \%$ (including complete response and partial response) 
in gemcitabine + taxane therapy, $100 \%$ partial response with ifosfamide-based therapy, and $50 \%$ partial response in CHOP-based therapy. The median duration of response with gemcitabine and docetaxel in particular was 13.4 months [9]. This study agreed with other cases that showed favorable outcomes with the combination of gemcitabine and docetaxel. Common factors in the patients who did not respond were bulky and/or intra-abdominal disease, as seen in our patient.

Pembrolizumab is a highly selective anti-programmed cell death-1 humanized monoclonal antibody which inhibits programmed cell death-1 (PD-1) activity by binding to the PD-1 receptor on T cells. Anti-PD-1 antibodies (including pembrolizumab) reverse T-cell suppression and induce antitumor responses [10]. In patients with undifferentiated pleomorphic sarcoma, pembrolizumab showed encouraging activity. In a phase 2, two-cohort, single-arm, open-label study, patients with soft tissue sarcoma or bone sarcoma were enrolled. Out of the 40 patients enrolled, 7 (18\%) had an objective response including 4 (40\%) of 10 patients with undifferentiated pleomorphic sarcoma and 2 (20\%) of 10 patients with liposarcoma (LPS). PD-L1 expression was not assessed in this study [11].

Pazopanib is a tyrosine kinase inhibitor that inhibits angiogenesis by inhibiting various cell surface receptors. It has been approved to treat renal cell carcinomas and soft tissue sarcomas, especially in patients who have advanced soft tissue sarcomas (aSTS) and have received prior chemotherapy. Several studies have shown pazopanib to be effective in treating aSTS. In a landmark phase 3 randomized study, the PALETTE trial, pazopanib was compared with placebo in patients with aSTS whose disease had progressed during or following prior chemotherapy. Longer median progression-free survival (mPFS) of 4.6 months (pazopanib) versus 1.6 months (placebo) was observed. The overall response rate was low at $6 \%$ for pazopanib versus $0 \%$ for placebo [12]. Another prospective single-arm phase 2 study showed further evidence of efficacy of pazopanib in LPS, leiomyosarcomas, synovial sarcomas, and an "other" group of less common sarcomas. Unfortunately, FDCS was not in this less common group. This multicenter study treated patients with intermediate or high-grade LPS with pazopanib and reported an mPFS of 4.4 months and an overall survival of 12.6 months [13]. These findings were consistent with the data for other STS subtypes that were studied in the PALETTE trial. There are ongoing studies to find predictive biomarkers for use in selecting patients with aSTS for pazopanib, but there is no clinically validated data at present. This unfortunately limits the clinical effectiveness and cost-effectiveness of the therapy [14].

In conclusion, there are several therapies that have been proven to be efficacious in treating aSTS. Because of the rarity of FDCS, however, there are still no treatment guidelines, and the data from therapies for aSTS must be extrapolated when treating FDCS. In the case presented above, chemotherapy-based treatments were initially given with progression of disease. Pazopanib, shown in several studies to have significant efficacy in advanced and aggressive soft tissue sarcomas, yielded significant regression of the tumor along with clinical improvement after just a few weeks of therapy. At the time of this writing, the patient had completed 9 months of therapy with pazopanib before showing progression on imaging studies. He was treated with sunitinib, ICE chemotherapy, and cabozantinib, unfortunately showing progression through all therapies. Hospice discussions are ongoing.

\section{Statement of Ethics}

The subject of the above case report has given their express permission in the form of written informed consent to publish their case, including publication of images.

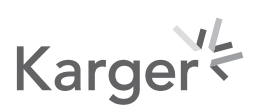




\section{Conflict of Interest Statement}

The authors have no conflicts of interest to declare.

\section{Funding Sources}

No funding sources were used in this case report.

\section{Author Contributions}

Pooja Shah - written contribution. Smit Shah - written contribution. Nicole Agostino access to case file, treatment modalities, written contribution.

\section{References}

1 Perez-Ordonez B, Erlandson RA, Rosai J. Follicular dendritic cell tumor: report of 13 additional cases of a distinctive entity. Am J Surg Pathol. 1996;20(8):944-55.

2 Wang RF, Han W, Qi L, Shan LH, Wang ZC, Wang LF. Extranodal follicular dendritic cell sarcoma: A clinicopathological report of four cases and a literature review. Oncol Lett. 2015;9(1):391-8.

3 Shahryari J, Ohgami RS. Follicular dendritic cell sarcoma. Atlas Genet Cytogenet Oncol Hematol. 2017;21(9): 324-6.

4 Dalia S, Shao H, Sagatys E, Cualing H, Sokol L. Dendritic cell and histiocytic neoplasms: biology, diagnosis, and treatment. Cancer Control. 2014;21(4):290-300.

5 Miyoshi R, Sonobe M, Miyamoto E, Date H. Completely resected follicular dendritic cell sarcoma of the posterior mediastinum: report of a case. Surg Case Rep. 2016;2(1):28.

6 Jain P, Milgrom SA, Patel KP, Nastoupil L, Fayad L, Wang M, et al. Characteristics, management, and outcomes of patients with follicular dendritic cell sarcoma. Br J Haematol. 2017 Aug;178(3):403-12.

7 Wang H, Su Z, Hu Z, Wen J, Liu B. Follicular dendritic cell sarcoma: a report of six cases and a review of the Chinese literature. Diagn Pathol. 2010;5(1):67-6.

8 Jain P, Patel KP, Futreal A, Gumbs C, Hu S, Bueso Ramos C, et al. Clinico-pathological characteristics, treatments and outcomes of patients with dendritic cell sarcoma (DS). Blood. 2015;126(23):2700.

9 Conry RM, Response of follicular dendritic cell sarcoma to gemcitabine and docetaxel: report of two cases and literature review. Clin Sarcoma Res. 2014 Jun 28;4:6.

10 Robert C, Ribas A, Wolchok JD, Hodi FS, Hamid O, Kefford R, et al. Anti-programmed-death-receptor-1 treatment with pembrolizumab in ipilimumab-refractory advanced melanoma: a randomised dosecomparison cohort of a phase 1 trial. Lancet. 2014;384(9948):1109-17.

11 Tawbi HA, Burgess M, Bolejack V, Van Tine BA, Schuetze SM, Hu J, et al. Pembrolizumab in advanced soft-tissue sarcoma and bone sarcoma (SARC028): a multicentre, two-cohort, single-arm, open-label, phase 2 trial. Lancet Oncol. 2017 Nov;18(11):1493-501.

12 Cesne AL, Bauer S, Demetri GD, Han G, Dezzani L, Ahmad Q, et al. Safety and efficacy of Pazopanib in advanced soft tissue sarcoma: PALETTE (EORTC 62072) subgroup analyses.BMC Cancer. 2019 Aug 13;19(1):794.

13 Sleijfer S, Ray-Coquard I, Papai Z, Le Cesne A, Scurr M, Schöffski P, et al. Pazopanib, a multikinase angiogenesis inhibitor, in patients with relapsed or refractory advanced soft tissue sarcoma: a phase II study from the European organisation for research and treatment of cancer-soft tissue and bone sarcoma group (EORTC study 62043). J Clin Oncol. 2009 Jul 1;27(19):3126-32.

14 Lee ATJ, Jones RL, Huang PH. Pazopanib in advanced soft tissue sarcomas. Signal Transduct Target Ther. 2019 May 17;4:16. 